\title{
APPLICATION OF CODED APERTURES IN TOMOGRAPHIC HEAD SCANNING
}

\author{
G.F. KNOLL, W.L. ROGERS, K.F. KORAL, J.A. STAMOS and N.H. CLINTHORNE \\ University of Michigan, Ann Arbor, MI 48109, USA
}

A circular ring tomograph, designated SPRINT, is under development for single photon emission tomography of the human head. Most data to date have been gathered using a single rotating slit inside the detector ring to define ray directions. As an alternative method, we have also investigated the use of multiplexed slit patterns consisting of a pseudorandom binary code. From theoretical considerations, one would expect that the substitution of the code for the single slit would have potential advantages in reducing statistical noise in tomographic images of small objects. It has been our experience that this improvement is marginal at best for the types of source distributions encountered in clinical brain imaging. However, the code shows substantial advantage in suppressing uncorrelated backgrounds and the effects of highly penetrating gamma ray components in the source.

\section{Introduction}

The application of coded aperture techniques in medical imaging began over ten years ago with the use of stationary apertures typified by the Fresnel zone plate [1-3] or multiple pinhole apertures [4,5]. A single coded image is recorded using these techniques, and is decoded using the unique spatial properties of the code. As an alternative, time coding techniques were also introduced $[6,7]$ in which a series of coded images are recorded over the course of a single measurement. Each of these images is taken through a unique spatial version of the code, and a single decoded image is then obtained using knowledge about the time modulation of the code and its autocorrelation function. The data from any typical detector element can be independently processed to derive a perspective view of the object, independent of all other detector elements.

An analysis of the noise behavior of images derived using time coding [6,8] shows several potential advantages over conventional non-coded imaging. Most of these derive from the different behavior of the decoded image with respect to photon statistical noise. In a conventional image, the statistical noise in a typical pixel depends only on the total number of detected photons in that same pixel. In coded images, the entire source distribution influences the statistical noise properties of all pixels. For portions of the source distribution that are more intense than the average across the field of view, this noise can be made lower than that obtained using non-coded techniques, while for other areas that are less intense than the average, the noise level is increased. For example, the intensity of a small object against a cold background in a coded image can be measured with greater statistical precision, but only at the expense of increased noise levels in the cold areas.
For broadly distributed source configurations, however, the use of codes with high transparency will result in higher statistical noise everywhere compared with conventional imaging.

Two aspects of the current application have motivated our interest in the use of time coding techniques. First, the application involves single photon emission tomography in which many separate images of the object taken from a full $360^{\circ}$ perspective are combined into a single reconstructed tomographic image. The projection process itself distributes noise from a single object point throughout the reconstructed image, and the effects of additional noise redistribution resulting from the coding process have not as yet been determined. Past studies of the signal-to-noise characteristics of coded apertures have generally assumed a planar source, while the present application involves a full transverse section tomographic reconstruction. As a second factor, we were also interested in suppressing the effects of high background levels found in some applications. For example, the decay scheme of pure ${ }^{123} \mathrm{I}$ involves the emission of approximately a $3 \%$ yield of high-energy gamma rays ranging from 180 to $780 \mathrm{keV}$ that are difficult to shield against and readily penetrate the septa of low-energy collimators. In addition, small amounts of ${ }^{124} I$ impurity in ${ }^{123} I$ produced by the $(p, 3 n)$ reaction can easily double the intensity of high-energy photons. While the yield of penetrating gamma rays from the region of interest may range from 3-6\%, most of the activity in a typical medical study is distributed widely throughout the patient's body. Therefore contributions of the high-energy gamma rays to the measured background can be orders of magnitude higher than expected from the region of interest alone. Many of them, by virtue of Compton escape, fall within the energy acceptance window of the imaging device. 


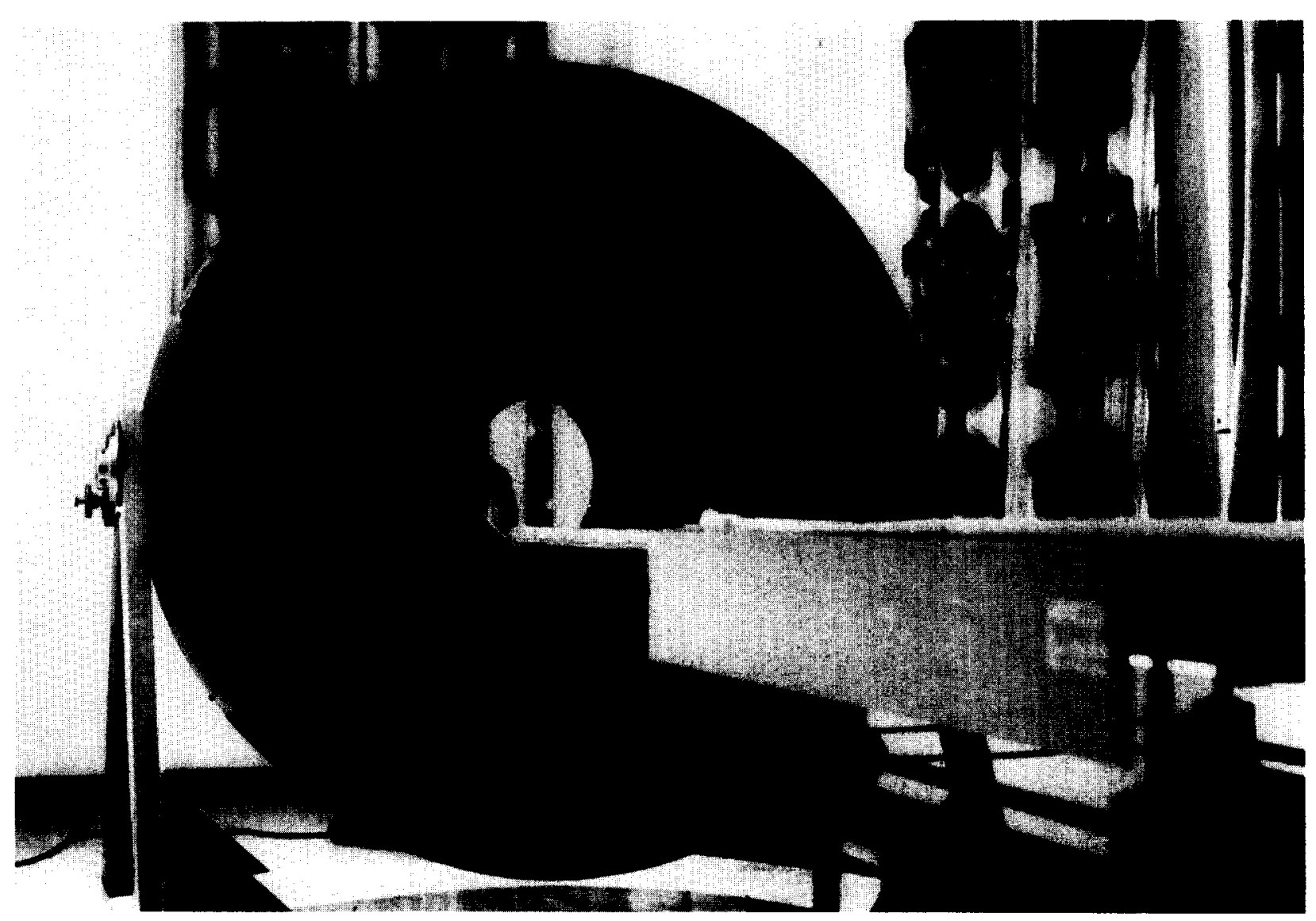

Fig. 1. Photograph of SPRINT oriented to produce transverse section tomographs of a supine patient.

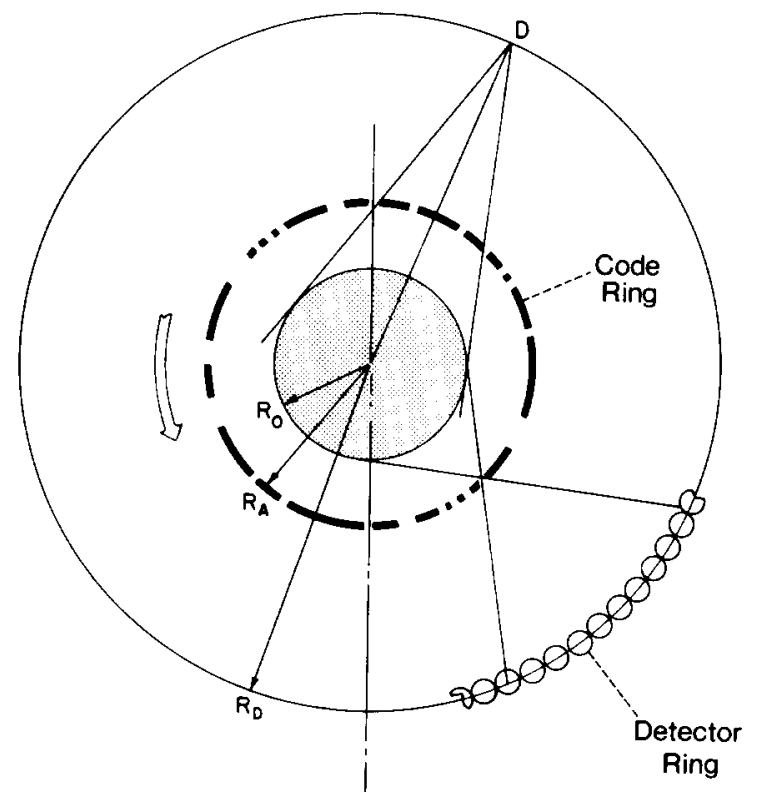

\section{Application to circular geometry}

We have implemented time coding techniques in the device known as SPRINT developed at the University of Michigan $[9,10]$. This instrument is designed for single photon emission tomography of the human head, and is based on a circular ring of 78 individual sodium iodide detectors arranged on a ring of $70 \mathrm{~cm}$ diameter. The completed device is illustrated in figs. 1 and 2. Each detector views the central source region through a rotating ring that is used to modulate the emitted gamma rays. Fig. 3 shows a photograph of the two separate rings employed in this study. Most of our previous data with SPRINT have been obtained using a simple nonmultiplexed ring in which 8 uniformly spaced pinholes are arranged around the circumference. Each pinhole is

Fig. 2. Diagram of the layout of SPRINT in the plane of the tomographic section. Radius of the detector circle $R_{\mathrm{D}}$ is $36 \mathrm{~cm}$, the aperture ring radius $R_{\mathrm{A}}$ is $16 \mathrm{~cm}$, and the field of view radius $R_{0}$ is $10 \mathrm{~cm}$. 


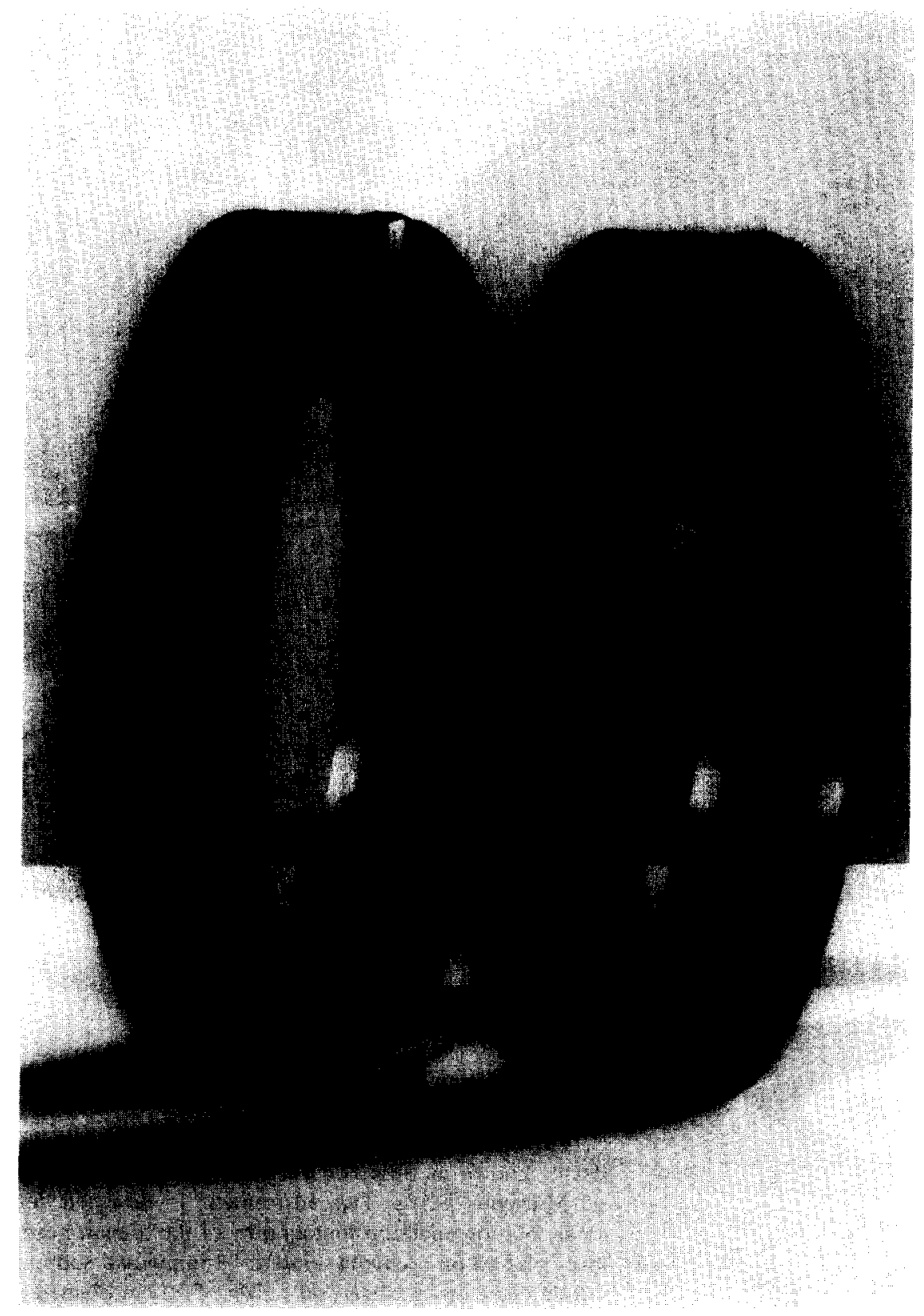

Fig. 3. Photograph of the two aperture rings used in this study: the 8-pinhole ring (left) and the coded ring (right).

in the form of an elongated slit perpendicular to the imaging plane. Conventional collimation restricts the vertical field of view of each detector to give an effeetive slice thickness of $1.0 \mathrm{~cm}$. Data are gathered by recording the counting rate as a function of time from each detector as the modulating ring is rotated. For the pinhole ring, the geometry is such that only one pinhole is in the field of view of a detector at any given time.
Thus, the position of the detector and the pinhole slit define a ray within the plane of interest that sweeps a complete fan beam view of the source as the ring is rotated through $1 / 8$ of a revolution. Each detector develops its own independent fan beam view of the object simultaneously, and these multiple views are then synthesized into a single tomogram using conventional reconstruction techniques. It is this "8-pinhole" ring 


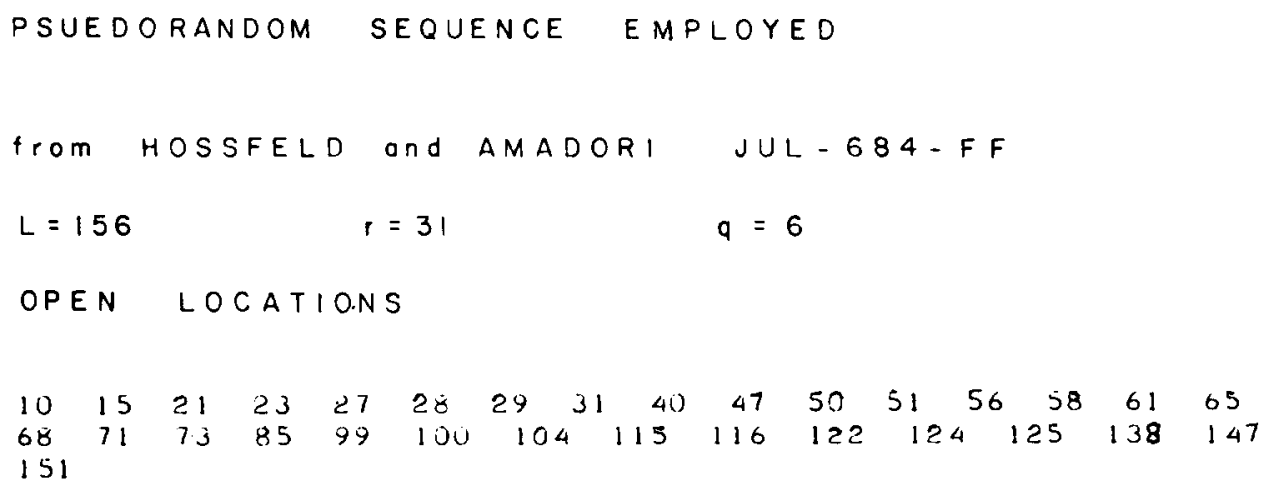
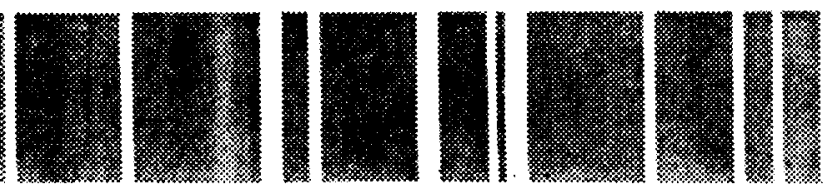

$\overline{0}$

Fig. 4. Diagram of the specific pseudorandom code sequence used in this study.

that has served in the present study as our conventional non-coded aperture for comparison purposes. This aperture is an optimal non-multiplexed aperture in the sense that all detectors view the $20 \mathrm{~cm}$ field of view throughout the measurement.

One feature of the design of SPRINT is the ease with which the modulating ring can be removed and replaced with another of different resolution or efficiency characteristics. To derive our coded images, the ring was replaced with the substitute shown on the right in fig. 3 . Here many slit pinholes are provided by a pattern of lead used to line the thin aluminum structure of the ring. The pattern of these slits is chosen to be the pseudorandom sequence illustrated in fig. 4. This pattern has 156 uniformly spaced locations, 31 of which represent an open slit. This pattern is distributed over one half the circumference of the coding ring, giving a width of $3.2 \mathrm{~mm}$ for each open slit. The same pattern is replicated on the second half of the ring. The code has an average transparency of $20 \%$, and an average of about 7 open slits are in the field of view of a typical detector at any given time. Data are now gathered by recording the counting rate from each detector as the ring is rotated through $1 / 2$ revolution. For any given detector, the gamma rays along any ray direction are thus modulated by the full sequence of open and closed slits. Each different ray direction is modulated by the same pattern but with a unique starting point or phase. Once all the data are recorded, the intensity of the source along each ray direction can be decoded by simply correlating the counting rate of the detector with the appropriate phase of the code [6].

The width of each individual element of the code is identical to the width of the pinhole slits in the ring used for comparison purposes, so the expected spatial resolution both with and without coding is the same. Previous studies [10] have shown this resolution to be $8.5 \mathrm{~mm}$ fwhm at the center of the field of view.

\section{Comparison images}

Data using either the coded or non-coded ring were recorded for each $1.6 \mathrm{~mm}$ increment of rotation of the ring circumference, giving two sample points across a typical slit width. Background was recorded by covering the open positions in the 8-pinhole ring with lead. These background counts were then subtracted from each recorded data set. Correlation calculations then provided separate fan beam data for each of the 78 individual detectors. These individual views were then synthesized into a tomographic image using the iterative reconstruction scheme CSIM [11] with attenuation corrections.

Two separate source phantoms were used in this study. The smaller consisted of a circular ring source with $2.0 \mathrm{~cm} \mathrm{ID} \mathrm{and} 5.0 \mathrm{~cm}$ OD. The larger was a Derenzo resolution phantom with overall diameter of 19 


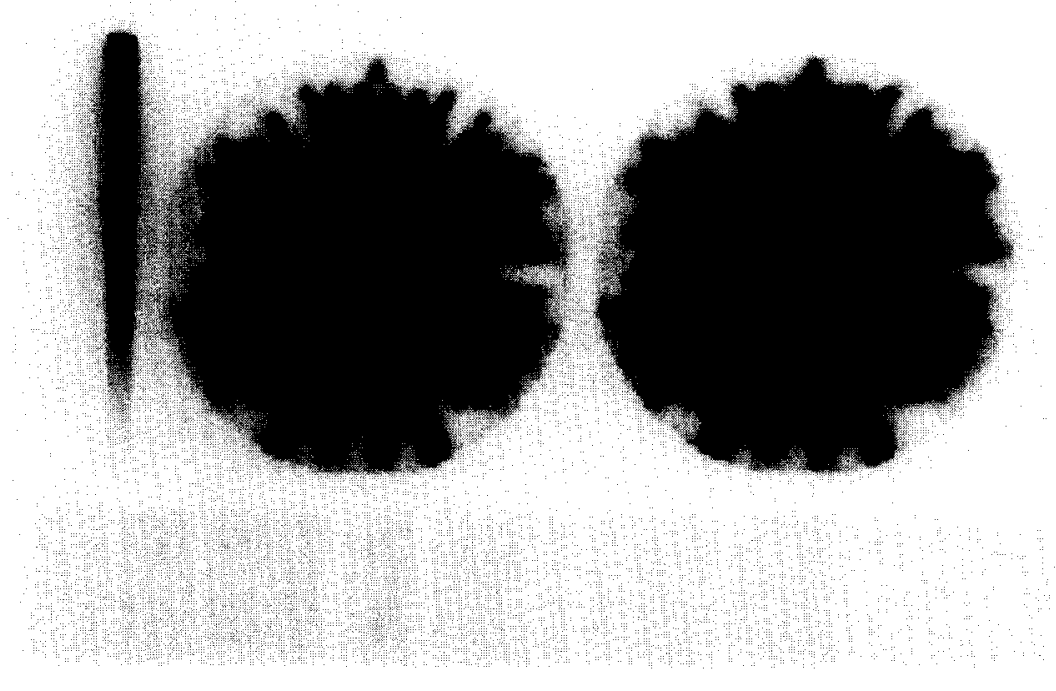

Fig. 5. Reconstructed images of the Derenzo resolution phantom: 8-pinhole (left), coded aperture (right).

$\mathrm{cm}$. In both cases the primary activity was provided by ${ }^{99 \mathrm{~m}} \mathrm{Tc}$, and pulse height windows in the detector electronics were set to bracket the $140 \mathrm{keV}$ gamma ray line. In order to simulate high-energy backgrounds, ${ }^{68} \mathrm{Ga}$ was later added to the smaller phantom to provide a source of $511 \mathrm{keV}$ annihilation radiation. The results are shown in the series of images in figs. 5 through 7.

\section{Discussion}

In each of the figures, an image pair is shown of the same object taken under identical conditions of source activity and imaging time. The conventional pinhole reconstruction is shown on the left, and the corresponding coded aperture reconstruction on the right.

Fig. 6. Reconstructed images of the ring phantom: 8-pinhole (left), coded aperture (right). Total activity of $2 \mathrm{mCi}$ with imaging time of $32 \mathrm{~s}$. 


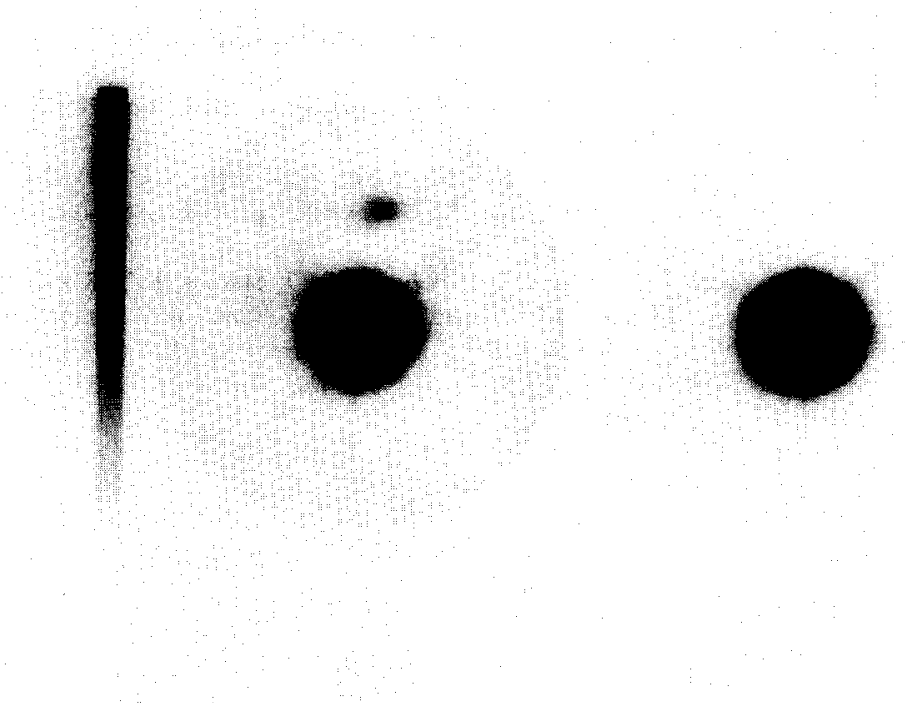

Fig. 7. Reconstructed images of the ring phantom in the presence of background: 8-pinhole (left), coded aperture (right). Same conditions as in fig. 6 , with the addition of ${ }^{68} \mathrm{Ga}$ source of $511 \mathrm{keV}$ source.

In fig. 5, the object consisted of a $7 \mathrm{~cm}$ thick Derenzo resolution phantom with a series of vertical tubes containing a total of $5 \mathrm{mCi}$ of ${ }^{99 \mathrm{~m}} \mathrm{Tc}$ activity. Imaging time was $800 \mathrm{~s}$ in both cases. Hole diameters in each hexagonal sector ranged from $6.2 \mathrm{~mm}$ for the largest to $2.5 \mathrm{~mm}$ for the smallest. Edge-to-edge hole spacing is 3 hole diameters in all cases. The signal-to-noise characteristics of both images are very similar, as would be expected in such a large, distributed object. The spatial resolution of the pinhole image is somewhat better, especially near the center of the reconstruction. We believe the difference is due to a small mechanical eccentricity of the coded ring, and is not a fundamental consequence of the coding. The comparison shows the capacity of the coded aperture to reconstruct objects with a large amount of internal structure and contrast.

The comparison of fig. 6 involves the smaller phantom for which the coding process would be expected to improve the noise properties of the reconstruction. Although not readily apparent from the gray-scale images, there is indeed an improvement in the smoothness with which the ring source is reconstructed. Circular profiles taken through both images show a reduced noise fluctuation in the coded image by almost a factor of two.

In fig. 7, the same object was modified by placing a vial of ${ }^{68} \mathrm{Ga}$ activity within the field of view. Pulse height windows remained set on the ${ }^{99 \mathrm{~m}} \mathrm{Tc}$ photopeak, so increased background resulted from a combination of Compton continuum in the detectors from the 511
$\mathrm{keV}$ annihilation photons and scattering of these photons in the phantom. Activity of the gallium source was chosen to result in a $1: 1$ background-to-signal counting rate when using the pinhole ring. This high-energy radiation is only slightly modulated by the aperture rings, so only a faint image of the gallium vial is visible in the upper half of the pinhole reconstruction. (The pinhole ring is made with thicker lead than the coded ring.) The additional noise introduced by this background is clearly evident in the pinhole image as fluctuations in the reconstruction that are broadly distributed across the entire field of view. There is a marked suppression of this noise for the coded reconstruction, to the point that it can no longer be easily perceived in the displayed images. The increased transparency of the code is known to strongly suppress uncorrelated background in conventional projection images [6], and we see here the translation of this advantage into the reconstructed tomogram.

\section{Conclusions}

The use of time coding in a tomographic device such as SPRINT may have its principal application in the suppression of image noise caused by uncorrelated background from high-energy gamma rays. While some improvement can be measured in the signal-to-noise properties of reconstructions of small objects in the absence of background, it is unlikely that application to the type of broadly distributed source configurations 
encountered in most medical imaging situations will result in significant improvement unless such uncorrelated background becomes comparable with the signal level. Nuclear medicine studies utilizing ${ }^{123} \mathrm{I}$ do involve substantial backgrounds and may provide applications in which coding can offer real advantage.

\section{References}

[1] H.H. Barrett, J. Nucl. Med. 13 (1972) 382.

[2] W.L. Rogers, K.S. Han, L.W. Jones et al., J. Nucl. Med. 13 (1972) 612

[3] M.D. Tipton, J.E. Dowdy, F.J. Bonte et al., Radiology 112 (1974) 155
[4] R.H. Dicke, Astrophys. J. 153 (1968) L101.

[5] L.T. Chang, S. Kaplan, B. MacDonald et al., J. Nucl. Med. 15 (1974) 1063.

[6] K.F. Koral, W.L. Rogers and G.F. Knoll, J. Nucl. Med. 16 (1975) 402.

[7] K.F. Koral and W.L. Rogers, Phys. Med. Biol. 24 (1979) 879.

[8] W.L. Rogers and R.S. Adler, Appl. Opt. 21 (1982) 324.

[9] G.F. Knoll and J.J. Williams, IEEE Trans. Nuc. Sci. NS-245 (1977) 581.

[10] W.L. Rogers, N.H. Clinthorne, J. Stamos, K.F. Koral, R. Mayans, J.W. Keyes, Jr., J.J. Williams, W.P. Snapp and G.F. Knoll, IEEE Trans. Med. Imaging MI-1 (1982) 63.

[11] W.P. Snapp, Ph.D. Dissertation, University of Michigan (1980). 